\title{
4 Neue Akteure: Sarmaten und andere
}

\begin{abstract}
Nun machten es die Amazonen zur Mittagszeit immer so. Sie zerstreuten sich, einzeln oder zu Zweien, um von einander abseits zur Notdurft zu gehen. Da Das die Scythen auch merkten, machten sie's eben so; und so machte sich Einer an Eine, die ganz allein war, und die Amazone sträubte sich nicht, sondern ließ sich's gefallen. Sprechen konnten sie nun zwar nicht, weil sie einander nicht verstanden, doch bedeutete sie ihn mit der Hand, des folgenden Tags wieder an den Ort zu kommen, und einen anderen mitzubringen; mit Zeichen, daß es zwey sein sollten, und daß auch sie eine andere mitbringen wolle. Der Jüngling ging also zurück und sagte das den Übrigen, und kam des anderen Tages mit noch Einem, wo er auch gleich die zweite Amazone schon wartend fand. Und die übrigen Jünglinge machten, da sie Das erfuhren, nun auch die übrigen Amazonen sich kirre. Hernach vereinigten sich ihre Lager, und wohnten beisammen und Jeder hatte die zum Weibe, zu der er sich zuerst gesellt habe. ${ }^{1}$
\end{abstract}

Aus diesen Verbindungen zwischen Skythen und Amazonen, so erzählt es zumindest Herodot, seien die Sauromaten hervorgegangen: „Und daher haben die Weiber der Sauromaten noch ihre alte Lebensart, gehen zu Pferde auf die Jagd, mit und ohne die Männer, gehen in den Krieg, und tragen auch denselben Anzug, wie ihre Männer.“2 Dies kann als Hinweis darauf gewertet werden, dass Frauen, wie in (halb-)nomadischen Gemeinschaften üblich, nicht auf die Lebensbereiche beschränkt waren, die hinlänglich als, weiblich' gelten - Haus, Hof, Familie etc. Die Frage, ob im Schwarzmeerraum und auf der Krim als Teil dessen Formen mutterrechtlicher, matrilokaler oder matriarchaler Gesellschaften beweisbar seien, wurde und wird immer wieder auch vor dem Hintergrund des Amazonen-Themas diskutiert. ${ }^{3}$ Mythos oder nicht, bei der Übertragung moderner Begriffe wie „Gleichstellung“ oder „Gleichberechtigung“ auf vergangene Zeiten ist grundsätzlich Vorsicht angezeigt. In jedem Fall gibt es aber valide archäologische Hinweise darauf, dass Frauen Waffen trugen und wohl auch an Kampfhandlungen teilnahmen. In Kurganen, in denen weibliche Überreste gefunden wurden, befanden sich häufig Waffen als Grabbeigaben. ${ }^{4}$ Das Amazonen-Bild ist auch deshalb von der Vorstellung waffentragender, kämpferischer Frauen geprägt. Die quasi-mythischen Amazonen seien, so heißt es beispielsweise in einem Arktinos von Milet (um 750 v.Chr.) zugeschriebenen epischen Gedicht, während des

1 Herodot, Historien, 4. Buch, 113, zitiert nach Schöll (1829), 501.

2 Herodot, Historien, 4. Buch, 116, zitiert nach Schöll (1829), 505.

3 Im 19. Jahrhundert erlangte insbesondere Johann Jakob Bachofens auf der Analyse antiker Mythen basierende Theorie von der sog. Gynaikokratie besondere Popularität: Bachofen (1997). Zur (Un-)Haltbarkeit der Beweiskraft der Grabungswissenschaften für die Existenz antiker Matriarchate vgl. die Positionen von Röder u.a. (1996).

4 Vgl. dazu Ascherson (1996), 177-186.

Ә OpenAccess. (C) 2020 Kerstin S. Jobst, publiziert von De Gruyter. (cc))BY Dieses Werk ist lizenziert unter der Creative Commons Attribution 4.0 International. https://doi.org/10.1515/9783110520620-006 
Troianischen Krieges vergeblich den Troianern zu Hilfe geeilt. Nach der Niederlage sei ihnen die Befreiung aus griechischer Gefangenschaft und die Flucht nach „Skythien“, also an das nördliche Schwarze Meer, gelungen. Die ,edle Abkunft“ der Amazonen und ihr Bezug zu einem der zentralen ,Ereignisse“ in der griechischen Mythologie erklärt, warum die Sarmaten/Sauromaten im Mythenbestand so vieler Nationen (vgl. Kapitel 2) eine besondere Rolle gespielt haben.

Die in vielen alten Texten in einem Atemzug genannten Begriffe „Sarmaten“ und „Sauromaten“ verwirren vermutlich und bedürfen einer Erklärung: In antiken Schriften wurden die Bezeichnungen „Sarmaten“ und „Sauromaten“ zum Teil different, häufig aber auch synonym benutzt. ${ }^{5}$ Mittlerweile wird der SarmatenBegriff zumeist als übergeordneter Terminus für verschiedene nomadische Stämme iranischer Herkunft aufgefasst; zu diesen zählen die Aorsen, Jazygen, Alanen, Roxolanen, Siraken und Maioten. ${ }^{6}$ Doch auch gegen diese Lesart werden Einwände angeführt, werden unter dem Terminus ,iranische Völker' doch ethnische Gruppen zusammengefasst, die indo-iranische Sprachen gesprochen haben. Einige WissenschaftlerInnen, darunter Anca Dan, weisen jedoch darauf hin, dass gar nicht bekannt ist, welche Sprache(n) die sog. Sauromaten/Sarmaten eigentlich gesprochen hätten, überliefern antike griechische Texte doch nur ein einziges unzweifelhaft sarmatisches Wort - „marha“, ein Schlachtruf. Mehr Beweiskraft für die iranische Herkunft der Sarmaten gebe hingegen ihr Name selbst, dessen Ursprung in der indo-iranischen Sprachfamilie plausibel sei. ${ }^{7}$ Zur weiteren Verwirrung trägt auch bei, dass antike Autoren häufig bei der Unterscheidung zwischen „Sarmaten“ und „Skythen“ in Konfusion gerieten: „Auch bei etlichen [...] Autoren der römischen Kaiserzeit werden in Exkursen immer wieder Skythen erwähnt, wobei mit diesem Begriff tatsächlich die Sarmaten gemeint sind. "8 Und der griechische Geograph Strabon (etwa 63 v.Chr. - 23 n.Chr.), der mit seinen berühmten „Erdbeschreibungen“ eine wichtige Quelle über die Sarmaten lieferte und deren siebtes Buch immer noch eine wichtige Quelle auch für die Geschichte der Krim ist, verwendet beide Termini häufig synonym. ${ }^{9}$

Wer genau nun diese Sarmaten waren, lässt sich nicht abschließend klären. Dan hat das ,Sarmaten-Problem“ treffend zusammengefasst: „,[O]ur current image of the Sarmatian is the result of loose readings of texts and archeological evid-

\footnotetext{
5 Lysenko (2006).

6 Grundlegend zu den Sarmaten vgl. Brzezinski u. Mielczarek (2002).

7 Dan (2017), 101. Diese Auffassung wird von anderen Autoren geteilt, vgl. z. B. Jankowski (2006), 25.

8 Parzinger (2004), 17.

9 Radt (2003).
} 
ence, nourished by nationalistic convictions. " ${ }^{10}$ Letztlich gilt dies aber nicht nur für die Sauromaten/Sarmaten oder für die bereits eingehender beschriebenen Skythen, sondern auch für viele andere Völkerschaften, die in dauernden oder vorübergehenden Kontakt mit der Krim traten. Die Forschung geht trotz mannigfacher Unklarheiten dennoch mehrheitlich davon aus, dass ab dem 3. Jahrhundert v.Chr. neue Akteure aus dem Osten auf die Krim vorstießen, welche vermutlich der indo-iranischen Sprachfamilie angehörten und die im Folgenden pragmatisch Sarmaten genannt werden. Bis zu ihrer Verdrängung durch bzw. Akkulturation mit den Goten (eine selbstverständlich ebenfalls eher unscharfe Sammelbezeichnung für ethnisch höchst heterogene Gruppen) im 4. Jahrhundert n. Chr. beeinflussten sie die Verhältnisse auf der Halbinsel nachhaltig. ${ }^{11}$

Dies zeigte sich u.a. daran, dass die Sarmaten die Skythen allmählich verdrängten bzw. sich mit diesen akkulturierten, was gegen das den sarmatischen Gruppen anhaftende Prädikat „barbarisch“ spricht, zumal es die Skythen doch zu beachtlichen kulturellen Veränderungen ihrer Lebensweise gebracht hatten: Ein Großteil hatte das Nomadentum aufgegeben, diverse stadtähnliche Anlagen gegründet und vor allem die Hauptstadt Neapolis (heute auf dem Gebiet Simferopol's gelegen) errichtet. Diese ist, wenn wir denn der offiziellen Homepage der Ausgrabungsstätte folgen, ,the biggest and the best investigated barbarian settlement of the North Pontic region. "12 Zwischen dem Ende des 3. Jahrhunderts v. Chr. bis in die zweite Hälfte des 3. Jahrhunderts n.Chr. existierte diese vom Skythen-Herrscher Skiluros gegründete Stadt, die aus der Steppe kommenden Bedrohungen lange Zeit standhielt, ehe die Goten ihr ein Ende bereiteten. Das skythische Reich ging über die eigentliche Halbinsel hinaus und reichte im Norden bis zum unteren Dnepr bei Olbia (nahe des heutigen Parutyne, Mykolajivs'ka oblast'). Auf der Krim selbst gelang u. a. die Einnahme der Chora von Chersones, also der landwirtschaftlich genutzten Gebiete der hellenischen Stadt. Deren Bevölkerung war, wie auf der Krim üblich, ethnisch gemischt. Archäologische Funde belegen den synkretischen kulturellen Charakter der Stadt; u.a. zeigen die griechischen architektonischen Elemente oder die Münzfunde mit griechischen Aufschriften den Einfluss des Hellenismus. Das baldige Auftauchen der Sarmaten bedeutete dann sowohl für die Skythen wie für die griechischen Kolonien der Krim eine veritable Bedrohung.

10 Dan (2017), 97.

11 Ajbabin (1999), 36, geht von einem Rückzug sarmatischer Gruppen in das südwestliche Berggebiet unterhalb des Berges Aj-Petri im 3. Jahrhundert n.Chr. aus.

12 Neapolis Scythian (2017). Vgl. dort auch weitere Informationen zu den Grabungen und Fotos der Anlage. Zur Geschichte der Stadt vgl. Zajcev (2003). 
